\title{
Mobile Automatic Detection System for Bacillus anthracis using Electrochemical DNA Chip
}

Jun Okada ${ }^{1 *}$, Hideki Horiuchi ${ }^{1}$, Koji Hashimoto ${ }^{1}$, Daiji Hirosawa ${ }^{1}$, Yohei Kurosaki' ${ }^{2,3}$, Keiko Kawamoto ${ }^{4}$, Jiro Yasuda ${ }^{2,3}$, Souichi Makino ${ }^{4}$, Nobuhiro Gemma ${ }^{1}$ and Masaru Nikaido ${ }^{1}$

${ }^{1}$ Toshiba Corporation, 1, Komukai-Toshiba-cho, Saiwai-ku, Kawasaki 212-8582, Japan

${ }^{2}$ National Research Institute of Police Science (NRIPS), 6-3-1, Kashiwanoha, Kashiwa 277-0882, Japan

${ }^{3}$ Nagasaki University, 1-12-4 Sakamoto, Nagasaki 852-8523, Japan

${ }^{4}$ Obihiro University of Agriculture and Veterinary Medicine (OUAVM), Inada-cho, Obihiro 080-8555, Japan

\begin{abstract}
A novel detection system for biological agents using Loop-Mediated Isothermal Amplification (LAMP) and an electrochemical DNA chip, Bio Bulwark ${ }^{\mathrm{TM}}$, has been developed. Genomic DNAs/RNAs roughly extracted from biological agents were amplified by the LAMP method using target-specific primers and strand-displace polymerase. The amplified DNAs were processed to hybridization with the electrochemical DNA chip. Anodic current derived from hoechst 33258, an electrochemically active intercalator, was relative to the amount of the target DNA hybridized with target-specific probes on the DNA chip. All reactions and measurements were conducted using disposable cassettes, which included the reagents for amplification and the DNA chip, and a mobile automatic detection system. The preliminary study showed that accurate detection of $B$. anthracis was carried out within 70 minutes. This system would prevent or minimize damage in the event of a criminal attack involving a biological threat.
\end{abstract}

Keywords: B. anthracis; Loop-mediated isothermal amplification; Mobile system; DNA chip cassette

\section{Introduction}

Rapid identification of biological warfare agents on site is important for preventing or minimizing damage in the event of a criminal attack involving a biological threat. Although various detection systems have been developed, there are few useful systems available at present in terms of sensitivity and plurality of detection targets [1-3].

Molecular techniques based on an enzymatic amplification such as polymerase chain reaction are powerful tool for the sensitive and specific detection of microorganisms. Recently Loop-Mediated Isothermal Amplification (LAMP) has developed and become popular due to high specificity, high sensitivity and easy handling [4,5]. There have been a growing number of reports on the detection of dangerous bacteria or viruses using the LAMP method [6-11]

A DNA chip is a powerful tool for detecting multiple genes simultaneously. Although DNA chips based on a fluorescence detection method are already in widespread use [12-15], they require complicated procedures for fluorochrome labelling. Furthermore, expensive and large optical equipment is necessary. Recently electrochemical detection methods have been developed to overcome the above-mentioned drawbacks [16]. It uses an electrochemically active intercalator as amplification or hybridization indicator and requires no labelling step. The electorochemical signal of intercalator is measured by micro electrode and small size potentiostat, therefore large and expensive equipment is unnecessary. A combination of LAMP and the electrochemical detection is reported to achieve a simple genetic testing [17-20].

Here we report a newly developed mobile system for the detection of biological agents, Bio Bulwark ${ }^{\mathrm{TM}}$, which performs the entire process from DNA amplification to detection automatically using the LAMP method and an electrochemical DNA chip.

\section{Materials and Methods}

\section{Pretreatment}

Environmental samples include impurities that inhibit DNA amplification and detection on the DNA chip. Furthermore, $B$. anthracis, a high-priority biological agent, exists as a spore in the field. The spore is a resistant form against unfavorable conditions such as high temperature, high $\mathrm{pH}$, dry condition and irradiation. The extraction of DNA from the spore is difficult because it has a very hard shell. We developed a pretreatment reagent kit for environmental samples to extract and purify the DNAs, which is based on the beads beating method [21]. The kit consists of a sampling tool (SWAB TEST Pro. media ST-25, Elmex Ltd.), zirconia-silica beads ( $\varphi 0.1 \mathrm{~mm}$, Tomy Medico Co., Ltd) for beads beating and isopropanol and ethanol for purification. First, powder, liquid or deposit samples are collected and suspended in a buffer solution using a sampling tool. The suspended samples are added to a bead beating tube to crush a cell. The tube is shaken vigorously using a mini bead beater (Biospec 3110BX) for 3 min. After centrifugation at room temperature, a supernatant is moved to another tube and heated at $80^{\circ} \mathrm{C}$ for $3 \mathrm{~min}$. Then, isopropanol and ethanol are added to the tube to precipitate and purify the DNA. We utilize B. subtilis as a sample in order to estimate extraction efficiency and purity of extracted DNA using the pretreatment kit, because it belongs to the same genus as $B$. anthracis and handling of the experiments is easier and safer. The extracted DNA was measured by a spectrophotometer.

\section{DNA amplification}

The reaction condition of LAMP for B. anthracis was as described in a previous paper [11]. The specific primers for three target genes

*Corresponding author: Jun Okada, Toshiba Corporation, 1, Komukai-Toshibacho, Saiwai-ku, Kawasaki 212-8582, Japan, Tel: +81-44-549-2044; Fax: +81-44549-2426; E-mail: jyun.okada@toshiba.co.jp

Received August 28, 2012; Accepted October 16, 2012; Published October 18 2012

Citation: Okada J, Horiuchi H, Hashimoto K, Hirosawa D, Kurosaki Y, et al. (2012) Mobile Automatic Detection System for Bacillus anthracis using Electrochemical DNA Chip. J Biosens Bioelectron 3:126. doi:10.4172/2155-6210.1000126

Copyright: ( 2012 Okada J, et al. This is an open-access article distributed under the terms of the Creative Commons Attribution License, which permits unrestricted use, distribution, and reproduction in any medium, provided the original author and source are credited. 
are designed: one is a unique gene in the genomic DNA (gDNA) and the other two are virulence plasmids, $\mathrm{pXO} 1$ and $\mathrm{pXO} 2$. The isothermal amplification was performed at $63^{\circ} \mathrm{C}$.

\section{DNA chip}

Figure 1 presents the principle of electrochemical DNA detection system [22]. The electrochemical DNA chip consists of gold electrodes patterned on glass substrate and DNA probes immobilized on electrodes. By applying target DNA on the DNA chip, the DNA probe with the complimentary sequence specifically hybridizes with the target DNA. After washing off the non-specifically bound DNAs, electrochemically active intercalator Hoechst 33258 is applied on the chip. The intercalator specifically binds to the double-stranded DNAs, i.e. hybridized DNA probes. When voltage is applied to the electrode, the intercalator is oxidized and emits electrons. Anodic current derived from Hoechst 33258 was relative to the amount of the target DNA hybridized with target specific probes on the electrode. Thus, the target DNA is detected simply by measuring an electrical current. DNA probes for three target genes of $B$. anthracis immobilized on the electrode surface were designed for a loop sequence of each LAMP product in accordance with a previous paper [23]. Oligonucleotide probes with a thiol group at 3' end were obtained as custom synthesis

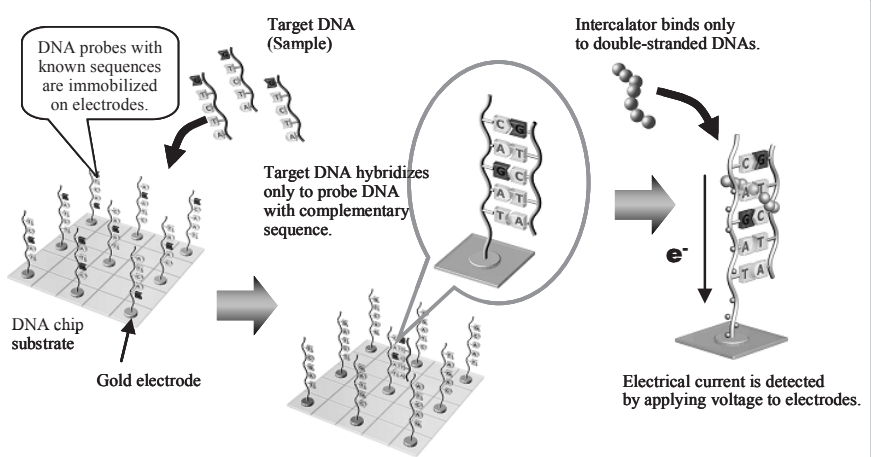

Figure 1: Detection principle of the electrochemical DNA chip.

(a)

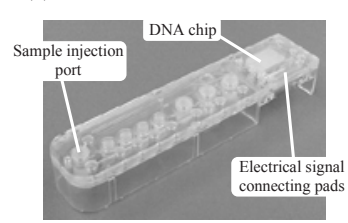

(b)

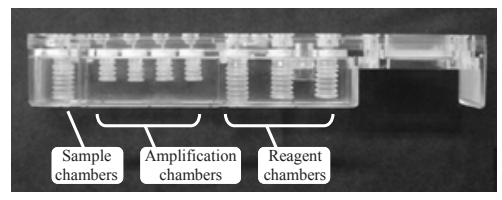

(c)

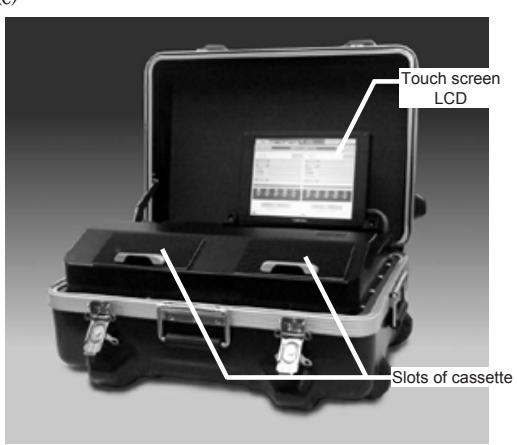

Figure 2: DNA chip cassette and automated DNA detection equipment Bio Bulwark ${ }^{\mathrm{TM}}$. products. Each working electrode was spotted with $0.1 \mu \mathrm{L}$ of the probe solution containing the $3 \mu \mathrm{M}$ oligonucleotide probes. Then, the chip was washed with distilled water and stored at $-20^{\circ} \mathrm{C}$. For each probe, four electrodes were assigned. The hybridization and washing reaction were controlled at 45 and $30^{\circ} \mathrm{C}$, respectively.

\section{DNA chip cassette}

The DNA chip cassette has functions for both DNA amplification by LAMP reaction and DNA detection by electrochemical DNA chip. As shown in (Figure 2a), on the top surface of the cassette, there is a sample injection port and electrical signal connecting pads. This cassette has liquid flow channels and accordion chambers that serves as the reagent reservoir and the pump, respectively. After injecting a sample into a cassette, the cassette is completely sealed and all reactions are thereafter performed within the airtight cassette in order to prevent contamination by leakage of amplified DNA products. There are eight chambers in the cassette as shown in (Figure 2b). The first chamber is to hold the injected sample (sample chamber), the next four chambers contain DNA primers and polymerase for multiplex amplification (amplification chambers), and the other chambers contain reagents for hybridization, washing, and electrochemical detection (reagent chambers). Sample and reagents flow through narrow channels in the cassette, and microvalves in the channels control the flow direction. All valves and pumps are controlled from outside. Figure 3 shows the schematic diagram of the sample and reagent flows and the reaction process in the cassette. An injected sample is divided into 4 amplification chambers that are heated for DNA amplification. All amplified DNA products are gathered into a mixing chamber and mixed with hybridization reagent stored in the chamber in advance. The mixed sample is transferred to the DNA chip and hybridized with DNA probes on the DNA chip. Then, a washing buffer removes non-specified hybridized DNA. Finally, intercalator molecules react with double-stranded DNA on the DNA chip and electrochemical measurement is performed. Apart from injection of the pretreatment sample, all processes were performed in the cassette without preparation of reagents.

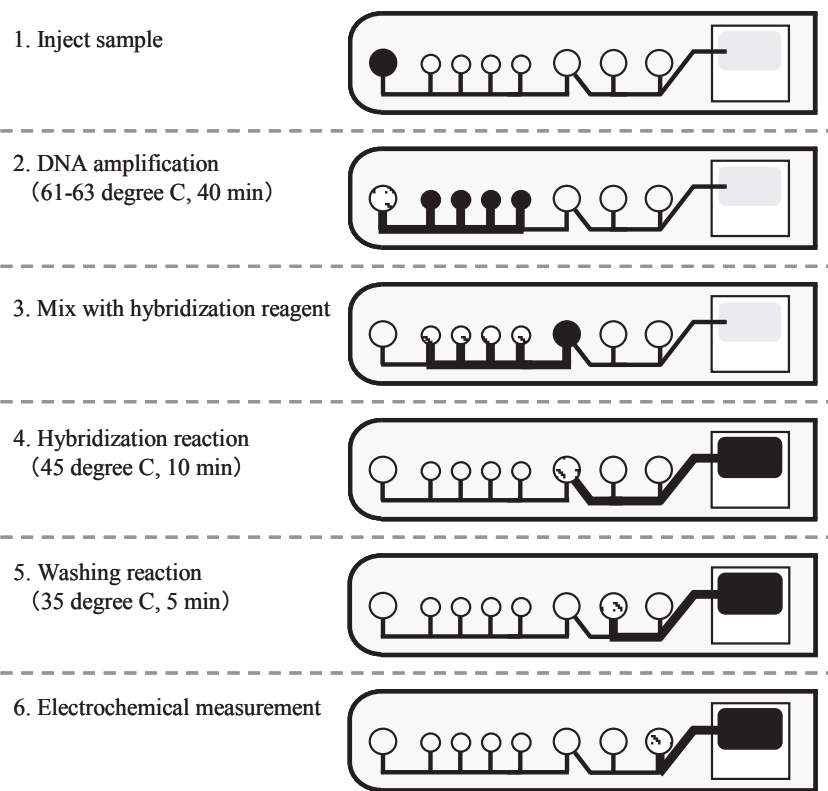

Figure 3: Processes of sample and reagents flow in the cassette. 
Citation: Okada J, Horiuchi H, Hashimoto K, Hirosawa D, Kurosaki Y, et al. (2012) Mobile Automatic Detection System for Bacillus anthracis using Electrochemical DNA Chip. J Biosens Bioelectron 3:126. doi:10.4172/2155-6210.1000126

Page 3 of 4

\section{Mobile fully automated DNA detection equipment}

Figure $2 \mathrm{c}$ shows the mobile fully automated DNA detection equipment Bio BulwarkTM for the DNA chip cassette described above. The entire system is incorporated in a compact and portable case. This equipment has a touch screen LCD for easy operation. A maximum of 15 kinds of biological agents are detectable simultaneously by using 2 cassette slots. Detection time is $70 \mathrm{~min}$ in high-sensitivity mode and 40 min in high-speed mode. All systems are controlled by a PC.

\section{Results and Discussion}

\section{Extraction of DNA from spores}

The extraction efficiency and purity of extracted DNA using the pretreatment kit are examined for $5 \times 108 \mathrm{cfu}$ of B. subtilis. The extraction efficiency of DNA was approximately $30 \%$, and the ratio of absorbance $(260 \mathrm{~nm} / 280 \mathrm{~nm})$ of extracted sample was around 2.0. These results indicated that the yield and purity of extracted DNA using the kit was sufficient for the molecular testing.

\section{Sensitivity of system}

After the extraction of DNA from B. anthracis using the pretreatment kit, we estimated the sensitivity of the Bio Bulwark system. Figure 4 shows a typical histogram of electrical signals for $B$. anthracis detection. Electrical signals from 3 genes related to $B$. anthracis are obviously higher than those from other genes. The dilution experiments showed that $10 \mathrm{pg}$ gDNA and $1 \mathrm{pg}$ pXO1 and $10 \mathrm{pg}$ pXO2 were clearly identified by using Bio Bulwark. A genome size of $B$. anthracis is about $5.23 \mathrm{Mbp}$ [24]. These amounts of genes are equivalent to $10^{\wedge} 3$ cfu bacteria.

\section{Specificity of system}

B. anthracis has different pathogenicity depending on the presence of plasmid DNA pXO1 and/or pXO2. By using various $B$. anthracis strains, we have checked the specificity of our DNA chip cassette. For Pasteur I and Pasteur II (No.30) strains that have both pXO1 and $\mathrm{pXO} 2$, the currents for all three probes are positive. For Davis strain and Pasteur II (No.34) with only pXO2, the current for pXO1 is negative. For Pasteur II (No.36) without plasmid, the signal of gDNA is only positive.

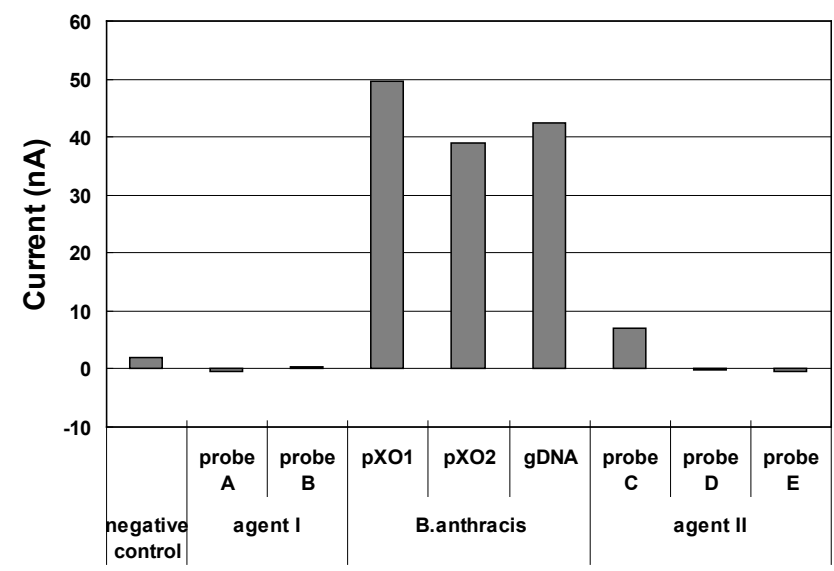

Figure 4: The histogram of electrical signals for B. anthracis (Pasteur I) detection.

pXO1, pXO2 and gDNA are DNA probe for $B$. anthracis. Probe $A$ and $B$ are DNA probe for other biological agent I. Probe $C, D$ and $E$ are DNA probe for other biological agent II.
We also tested cross-reactivity to 12 species (23 strains) closely related to $B$. anthracis, such as B. cereus and other Bacillus spp. The electrochemical currents for all 3 genes are negative to the related species. These results proved the high specificity for various anthrax strains.

Although we only describe the results of $B$. anthracis detection in this paper, the DNA chip cassette was not limited to B. anthracis. We have accomplished the detection of nineteen biological agents using this system.

\section{Conclusion}

We have developed a mobile system for biological agent detection, Bio Bulwark ${ }^{\mathrm{TM}}$. Only by injecting a crude extract into a disposable cassette, the target genes of $B$. anthracis were easily detected within 70 min. This system makes it possible to detect biological agents on site with high sensitivity, specificity and rapidity. It is expected that this system would prevent or minimize damage in the event of a criminal attack involving a biological threat.

\section{Acknowledgment}

A part of this work is supported by a grant from the Core Research for Evolution Science and Technology (CREST), Project of Japan Science and Technology Agency (JST).

\section{References}

1. King D, Luna V, Cannons A, Cattani J, Amuso P (2003) Performance assessment of three commercial assays for direct detection of Bacillus anthracis spores. J Clin Microbiol 41: 3454-3455

2. Lim DV, Simpson JM, Kearns EA, Kramer MF (2005) Current and developing technologies for monitoring agents of bioterrorism and biowarfare. Clin Microbiol Rev 18: 583-607.

3. Christensen DR, Hartman LJ, Loveless BM, Frye MS, Shipley MA, et al. (2006) Detection of biological threat agents by real-time PCR: comparison of assay performance on the R.A.P.I.D., the LightCycler, and the Smart Cycler platforms. Clin Chem 52: 141-145.

4. Notomi T, Okayama H, Masubuchi H, Yonekawa T, Watanabe K, et al. (2000) Loop-mediated isothermal amplification of DNA. Nucleic Acids Res 28: E63.

5. Iwamoto T, Sonobe T, Hayashi K (2003) Loop-mediated isothermal amplification for direct detection of Mycobacterium tuberculosis complex, M. avium, and $M$. intracellulare in sputum samples. J Clin Microbiol 41: 2616-2622.

6. Fukuma A, Kurosaki Y, Morikawa Y, Grolla A, Feldmann H, et al. (2011) Rapid detection of Lassa virus by reverse transcription-loop-mediated isothermal amplification. Microbiol Immunol 55: 44-50.

7. Kurosaki Y, Takada A, Ebihara H, Grolla A, Kamo N, et al. (2007) Rapid and simple detection of Ebola virus by reverse transcription-loop-mediated isothermal amplification. J Virol Methods 141: 78-83.

8. Kurosaki Y, Grolla A, Fukuma A, Feldmann H, Yasuda J (2010) Development and evaluation of a simple assay for Marburg virus detection using a reverse transcription-loop-mediated isothermal amplification method. J Clin Microbio 48: $2330-2336$

9. Ohtsuki R, Kawamoto K, Kato Y, Shah MM, Ezaki T, et al. (2008) Rapid detection of Brucella spp. by the loop-mediated isothermal amplification method. J App Microbiol 104: 1815-1823.

10. Sakuma T, Kurosaki Y, Fujinami Y, Takizawa T, Yasuda J (2009) Rapid and simple detection of Clostridium botulinum types A and B by loop-mediated isothermal amplification. J Appl Microbiol 106: 1252-1259.

11. Kurosaki Y, Sakuma T, Fukuma A, Fujinami Y, Kawamoto K, et al. (2009) A simple and sensitive method for detection of Bacillus anthracis by loopmediated isothermal amplification. J Appl Microbiol 107: 1947-1956.

12. Pease AC, Solas D, Sullivan EJ, Cronin MT, Holmes CP, et al. (1994) Lightgenerated oligonucleotide arrays for rapid DNA sequence analysis. Proc Nat Acad Sci U S A 91: 5022-5026.

13. Ramsay G (1998) DNA chips: state-of-the art. Nat Biotechnol 16: 40-44. 
Citation: Okada J, Horiuchi H, Hashimoto K, Hirosawa D, Kurosaki Y, et al. (2012) Mobile Automatic Detection System for Bacillus anthracis using Electrochemical DNA Chip. J Biosens Bioelectron 3:126. doi:10.4172/2155-6210.1000126

14. Dufva M (2009) Introduction to microarray technology. Methods Mol Biol 529: $1-22$.

15. Sanoudou D, Mountzios G, Arvanitis DA, Pectasides D (2012) Arraybased pharmacogenomics of molecular-targeted therapies in oncology. Pharmacogenomics J 12: 185-196.

16. Hashimoto K, Ito K, Ishimori Y (1994) Sequence-specific gene detection with a gold electrode modified with DNA probes and an electrochemically active dye. Anal Chem 66: 3830-3833.

17. Hongo S, Okada J, Hashimoto K, Tsuji K, Nikaido M, et al. (2008) Development of an automated DNA detection system using an electrochemical DNA chip technology. J Control Meas Syst Integr 1: 265-270.

18. Nakamura N, Ito K, Takahashi M, Hongo S, Hashimoto K, et al. (2009) Clinical verification of a combination technology of a loop-mediated isothermal amplification method and an electrochemical DNA chip for personalized medicine. Clin Biochem 42: 1158-1161.

19. Nagatani N, Yamanaka K, Saito M, Koketsu R, Sasaki T, et al. (2011) Semireal time electrochemical monitoring for influenza virus RNA by reverse transcription loop-mediated isothermal amplification using a USB powered portable potentiostat. Analyst 136: 5143-5150.
20. Hsieh K, Patterson AS, Ferguson BS, Plaxco KW, Soh HT (2012) Rapid sensitive, and quantitative detection of pathogenic DNA at the point of care through microfluidic electrochemical quantitative loop-mediated isothermal amplification. Angew Chem Int Ed Engl 51: 4896-4900.

21. Kurosaki Y, Horiuchi H, Fujinami Y, Hashimoto K, Yasuda J (2010) Development and application of a rapid and simple method for extrcting nucleic acids from microbes. Jpn J Forens Sci Technol 15: 135-142.

22. Goto K, Horiuchi H, Shinohara H, Motegi K, Hashimoto K, et al. (2007) Specific and quantitative detection of PCR products from Clostridium piliforme, Helicobacter bilis, $H$. hepaticus, and mouse hepatitis virus infected mouse samples using a newly developed electrochemical DNA chip. J Microbiol Methods 69: 93-99.

23. Nakamura N, Ito K, Hashimoto M, Nakamura A, Hayashimoto N, et al. (2011) Development of multisample detection system using a tag insertion primer and an electrochemical DNA chip. Anal Biochem 419: 190-195.

24. Read TD, Peterson SN, Tourasse N, Baillie LW, Paulsen IT, et al. (2003) The genome sequence of Bacillus anthracis Ames and comparison to closely related bacteria. Nature 423: 81-86. 